\title{
Development and validation of a multiplex-PCR assay for X-linked intellectual disability
}

Paula Jorge ${ }^{1 * \dagger}$, Bárbara Oliveira ${ }^{1,2+}$, Isabel Marques ${ }^{1}$ and Rosário Santos ${ }^{1}$

\begin{abstract}
Background: X-linked intellectual disability is a common cause of inherited cognitive deficit affecting mostly males. There are several genetic causes implicated in this condition, which has hampered the establishment of an accurate diagnosis. We developed a multiplex-PCR assay for the mutational hotspot regions of the FMR1, AFF2 and ARX genes.

Methods: The multiplex-PCR was validated in a cohort of 100 males selected to include known alleles for the FMR1 repetitive region: five full mutations (250-650 CGGs), ten premutations (70-165 CGGs) and eighty-five in the normal range (19-42 CGGs). Sequencing or Southern blotting was used to confirm the results, depending on the allele class. In this cohort, with the exception of one sample showing an AFF2 intermediate-sized allele, all other samples were normal (8-34 CCGs). No ARX variant was found besides the c.429_452dup. The validated assay was applied to 5000 samples (64.4\% males and $35.6 \%$ females).
\end{abstract}

Results: The normal-allelic range of both FMR1 and AFF2 genes as well as the nature of ARX variants identified was similar in both genders. The rate of homozygosity observed in female samples, $27.5 \%$ for FMR1 and 17.8\% for AFF2 alleles, is comparable to that published by others. Two FMR1 premutations were identified, in a male (58 CGGs) and a female case $\left[(C G G)_{47} /(C G G)_{61}\right]$, as well as several FMR1 or AFF2 intermediate-sized alleles. One AFF2 premutation (68 CCGs) and two putative full expansions were picked up in male subjects, which seems relevant considering the rarity of reported AFF2 mutations found in the absence of a family history.

Conclusions: We developed a robust multiplex-PCR that can be used to screen the mutational hotspot regions of FMR1, AFF2 and ARX genes. Moreover, this strategy led to the identification of variants in all three genes, representing not only an improvement in allele-sizing but also in achieving a differential diagnosis. Although the distinction between females who are truly homozygous and those with a second pre- or full mutation sized allele, as well as a definitive diagnosis, requires a specific downstream technique, the use of this multiplex-PCR for initial screening is a cost-effective approach which widens the scope of detection.

Keywords: X-linked intellectual disability (XLID), FMR1, AFF2, ARX, Multiplex-PCR

\section{Background}

$\mathrm{X}$-linked intellectual disability (XLID) is a group of genetically heterogeneous disorders caused by mutations in Xchromosome genes. Currently, it is believed that X-linked recessive gene defects account for about $10-12 \%$ of overall intellectual disability (ID) seen in males [1]. The lack of a comprehensible relationship between clinical signs and genotype in XLID, together with the large number of

\footnotetext{
* Correspondence: jorgpaula@gmail.com

${ }^{\dagger}$ Equal contributors

${ }^{1}$ Centro de Genética Médica Dr. Jacinto Magalhães, CHP, Praça Pedro Nunes 88, 4099-028, Porto, Portugal

Full list of author information is available at the end of the article
}

genes implicated, are the major obstacles in their genetic diagnosis and places ID as one of the most important unsolved problems in healthcare. Nowadays, a huge amount of information can be generated by assessing ID from both the clinical and molecular point of view. However, the challenge remains as to how all of this information can be used to establish a simple, standard and effective strategy applicable to molecular diagnosis and genetic counselling. Among the genetic causes involved in XLID, mutations in the genes FMR1 (fragile X mental retardation 1) and ARX (aristaless related homeobox) emerge as important causes. AFF2 (AF4/FMR2 family, member 2) testing is often

\section{Biomed Central}


requested when patients test negative for $F M R 1$, particularly in the absence of specific syndromic features [2-4].

The FMR1 gene contains polymorphic repetitive regions susceptible to suffer dynamic mutations, a process that may give rise to pathogenic expansions. The expansion to over 200 CGG repeats in the FMR1 gene is associated with fragile X syndrome [FXS, FRAXA; MIM\#300624], the most common form of familial severe XLID [5,6]. Below 200 repeats, alleles are classified as normal (5-44 CGGs), intermediate (45-54 CGGs) or premutated (55200 CGGs) (see also the Technical Standard and Guideline for Fragile X Testing, http://www.acmg.net/Pages/ ACMG_Activities/stds-2002/fx.htm, 2006 edition, last accessed September 2012) [7].

Besides the X-chromosome fragile site A, fragile site E (locus in Xq28) is also associated with intellectual disability [FRAXE; MIM \#309548]. This disorder is mainly a non-syndromic form of X-linked intellectual disability and is the result of silencing of the $A F F 2$ gene, as a consequence of an upstream CCG expansion [8]. In the normal population, the number of CCG repeats varies between 6 and 35, while it is increased to more than 200 hyper-methylated triplets in FRAXE/AFF2 intellectually disabled patients. In contrast to FXS, reports of $A F F 2$ full expansions are very rare and the dynamics of this repeat is not as clearly characterized as that of FMR1. Indeed, to what extent the alleles with CCG repeats in the range between 36 and 199 may exhibit a pathogenic role remains elusive, in part due to the rarity of the mutation and extended pedigrees [9].

In the $A R X$ gene, the second exon represents a mutational hotspot as it contains repetitive regions that codify alanines. Since its identification, several mutations have been identified, including insertions, deletions, duplications, missense, nonsense and splice mutations, leading to a wide spectrum of phenotypes that include syndromic as well as nonsyndromic forms of intellectual disability [10]. These different mutations were reported in patients with over 10 distinct clinical outcomes. Even though the mutations described extend across the gene, the majority occur in the largest coding exon of the $A R X$ gene, exon 2, where variations in polyalanine tracts, are among the most frequent $[11,12]$. Indeed the c.429_452dup mutation, affecting the second tract, is the most frequent $A R X$ mutation [13]. The absence of genotype/phenotype correlation in the $A R X$ gene explains our inability to establish the respective guidelines for molecular screening particularly in XLID patients that do not include brain malformations.

As the early recognition of syndromic physical traits is difficult, it is widely accepted that boys with unexplained ID/developmental delay, with or without any other clinical findings, should be screened for FXS. The literature supports the benefit of a screening method due to its prevalence and the fact that molecular genetic testing for FXS is highly sensitive and specific [14]. To the best of our knowledge there is no reference to a three-gene approach to the diagnosis of ID, specifically one that covers two of the most frequently mutated XLID genes. To address this issue we have developed a molecular test based on a multiplex-PCR, focusing on mutational hotspots of FMR1, AFF2 and ARX genes and validated in a male cohort. Using this new assay we screened 5000 samples from male and female subjects referred for FXS testing and have widened the identification of the genetic causes of some XLID cases.

\section{Methods}

All genomic DNA (gDNA) samples had previously been extracted by the salting out method, from peripheral blood collected in ethylenediamine tetra acetic acid (EDTA), dissolved in Tris-EDTA buffer $(\mathrm{pH}=7)$ and stored at $4^{\circ} \mathrm{C}$ [15]. No patients were recruited specifically for this study; all samples belong to subjects previously referred by medical geneticists for fragile X syndrome (FXS) molecular testing [16]. Their phenotypic spectrum ranged from severe intellectual disability to language delay or learning disabilities with normal to borderline psychomotor development. Approval for this study was obtained from Ethics committee of the Instituto Nacional de Saúde Dr. Ricardo Jorge (INSA, I.P.). The participants, or their legally authorized representatives, had given informed consent to take part in this research.

A validation cohort consisting of 100 male gDNAs was chosen to include normal FMR1 alleles, ten FMR1 premutations and five full mutations. A sample with the ARX mutation, c.429_452dup, was also used. This mutation had previously been published and described as c.428_451dup24 [17]. The test group comprised 5000 gDNAs of which $64.4 \%(\mathrm{n}=3220)$ were males and $35.6 \%$ $(\mathrm{n}=1780)$ females.

The method developed consisted in the combination of three primer pairs that amplify target regions, as follows: FMR1, forward 6-FAM ${ }^{\mathrm{mm}}$ labelled 5' CCA TCT TCT CTT CAG CCC TGC 3' and reverse 5' TTC GGT TTC ACT TCC GGT G 3'; AFF2, forward HEX labelled 5' TGT GAG TGT GTA AGT GTG TGA TGC TGC C 3' and reverse 5' CCG CGC GCA CCC AGC GAC $3^{\prime}$ and $A R X$, forward $5^{\prime}$ CAG CAG CCC TGG CTG GGA CTC $3^{\prime}$ and reverse HEX labelled 5' CGG TAC GAC TTG CTG CGG CTG 3'. While FMR1 and AFF2 amplicons encompass the CGG and CCG polymorphic triplet repeats, respectively, the $A R X$ exon 2 PCR (ARX ex2p), is designed to amplify a portion of the second exon of $A R X$ gene, that includes two of the three stretches of repetitive alanine coding triplets (poly AI and AII); a 380 bp amplicon corresponding to the normal allele is produced 
or, in the presence of the c.429_452dup, a 404 bp amplicon. First, the optimization process was done using each pair of primers in a separate amplification reaction and tested for annealing temperatures ranging from $55^{\circ} \mathrm{C}$ to $62^{\circ} \mathrm{C}$. PCR components were then tested in a multiplexed reaction, one by one, in an empirically decided order, namely primer amounts, extension time and concentration of several PCR co-adjuvants such as 7-deaza dGTP, betaine and DMSO. The optimized multiplex-PCR mixture prepared in a final volume of $25 \mu \mathrm{L}$ contained a final concentration of $1 \mathrm{M}$ betaine, $0.12 \mathrm{mM}$ each dATP, dCTP and dTTP (Bioline, London, UK), $0.02 \mathrm{mM}$ dGTP (Bioline, London, UK), $0.4 \mathrm{mM}$ 7-deaza dGTP, Li-Salt (Roche, Indianapolis, USA), 4.8\% DMSO (Sigma-Aldrich Co, St. Louis, USA), 10 pmol FMR1 primers, 6 pmol $A F F 2$ primers, 5 pmol $A R X$ primers and $1 \times$ Accutaq buffer (Sigma-Aldrich Co, St. Louis, USA). This mixture was supplemented with $1 \mathrm{U}$ AccuTaq polymerase (Sigma-Aldrich Co, St.Louis, USA) and a total of $300 \mathrm{ng}$ of gDNA was added at the end. The amplification reaction was performed in a 9800 Fast Thermal Cycler (Applied Biosystems, Foster City, CA, USA). After an initial denaturation step for 10 minutes at $98^{\circ} \mathrm{C}, 42$ cycles of amplification were performed as follows: denaturation at $98^{\circ} \mathrm{C}$ for 45 seconds, annealing at $58^{\circ} \mathrm{C}$ for 45 seconds and extension at $68^{\circ} \mathrm{C}$ for 2 minutes and 30 seconds, followed by an additional cycle of 10 minutes at $68^{\circ} \mathrm{C}$ for the final extension. PCR products were diluted at $1: 15$ in a reaction solution containing $0.5 \mu \mathrm{L}$ GeneScan $^{\text {Ts }}$ 500 ROX or MapMarker-1000-ROX, MapMarker ${ }^{\circledR}$ Size Std (BioVentures, Inc., Murfreesboro, TN, USA) and 13.5 $\mu \mathrm{L} \mathrm{Hi-Di} \mathrm{formamide} \mathrm{(Applied} \mathrm{Biosystems,} \mathrm{Foster} \mathrm{City,}$ CA, USA). Fragments were resolved on the $3130 \times 1$ Genetic Analyser and further analysed using the GeneMapper ${ }^{\circ}$ Software v4.0 (Applied Biosystems, Foster City, CA, USA).

Two types of methodologies were used to confirm the results obtained in the validation cohort, according to the allele class: sanger dideoxy sequencing for the amplifiable fragments, using primer sets upstream and downstream the FMR1 CGG, or AFF2 CCG repetitive regions, or the $A R X$ ex $2 \mathrm{p}$, and Southern blotting, for the non-amplifiable fragments or alleles that fall into the pathogenic range. Sequencing was performed using BigDye ${ }^{\circledast}$ Terminator v1.1 Cycle Sequencing Kit (Applied Biosystems, Foster City, CA, USA) following the manufacturer's indications with the primers described above (without the fluorescent labelling) and standard amplification conditions. Sequencing reactions were resolved on a $3130 \times 1$ Genetic Analyser. The resulting data were further analysed using the SeqScape ${ }^{\bullet}$ Software v2.5 (Applied Biosystems, Foster City, CA, USA). Southern blot genotyping was done using $\sim 10 \mu \mathrm{g}$ of gDNA double-digested with the restriction enzymes EcoRI and EagI for FMR1 gene or AflIII and NotI for AFF2 gene
(New England Biolabs, Ipswich, MA, USA). Further, the digested gDNA products were electrophoresed in parallel with a 1:1 mixture of the standard size markers, DNA Molecular Weight Marker II and III digoxigenin-labeled (Roche Applied Science, Indianapolis, IN, USA), blotted and hybridized using the FMR1 or the AFF2 digoxigeninlabeled specific probes, GLFXDIG1 or AJ31Dig1, as appropriate (Gene Link ${ }^{\mathrm{TM}}$, Hawthorne, NY, USA).

\section{Results and discussion}

A screening strategy is described that allows the characterization of FMR1 and AFF2 repetitive regions in normal to premutation ranges together with the detection of size variants in the second exon of $A R X$ gene. The amplification of FMR1 CGG repetitive region has been widely tested for several PCR conditions, either changing polymerase or adding co-adjuvants [18-21]. Due to the high GC content in all three amplified fragments and particularly in the repetitive amplified regions (of FMR1 and AFF2 genes) leading to stutter band formation, a directly comparative quantitative result was not consistently obtained. Instead, we observed reduction in stutter band formation particularly in FMR1 alleles, in amplifications using this multiplex-PCR, when compared to simplex reactions. Moreover, the simultaneous amplification of two similar fragments (FMR1 and $A F F 2$ polymorphic regions), in terms of GC content and presence of triplet repeats, provides an internal amplification control.

The validation cohort consisting of 100 male gDNAs was sequenced across the FMR1 and AFF2 repetitive regions and the $A R X$ ex $2 \mathrm{p}$, used for allele number standardization for each gene and for further validation of the multiplex-PCR methodology. Table 1 compiles these results. Examples of the optimized multiplex-PCR, sequencing and Southern blot results are shown in Figures 1 and 2. Overall, FMR1 CGG repeat sizes ranged from 19 to over 120, where the 30 CGG repeat allele represented 37\% of the observed alleles. AFF2 CCG repeat sizes ranged from 8 to 47 and alleles with 14 triplets represented $36 \%$ of the pool [Figure 1-I(a) and II(b), Figure 2(f)]. Besides, no other $A R X$ sequence variant was observed [Figure 1-I (b) and II(c)].

Samples with premutations and full mutations were on purpose included in the validation process to test for FMR1 primer specificity in the multiplex-PCR assay [Figure 1-I(c)]. Expanded alleles were not the main purpose of this assay and presented inconsistent amplification results. Thus, the 110 CGG allele was settled as the reliable upper-boundary for the sequencing validation [Figure 1-II(a), Figure 2(b)]. Furthermore, whenever the multiplex-PCR assay revealed either absence or an expanded allele, the corresponding sample was subjected to Southern blot analysis. An obvious gain when using 
Table 1 Validation cohort results

\begin{tabular}{|c|c|c|c|c|c|c|c|c|c|c|c|c|}
\hline$n$ & FMR1 CGG & AFF2 CCG & $A R X(\mathrm{bp})$ & $n$ & FMR1 CGG & AFF2 CCG & $A R X$ (bp) & $n$ & FMR1 (CGG) & AFF2 (CCG) & $A R X(\mathrm{bp})$ & $\begin{array}{c}\text { Obtained/confirmed by } \\
\text { Southern bloting }\end{array}$ \\
\hline 1 & 19 & 13 & 380 & 3 & 30 & 11 & 380 & 1 & 33 & 22 & 380 & \\
\hline $1^{a}$ & 19 & 47 & 380 & 4 & 30 & 13 & 380 & 1 & 35 & 34 & 380 & \\
\hline 2 & 20 & 14 & 380 & $1^{b}$ & 30 & 14 & 404 & 2 & 36 & 14 & 380 & \\
\hline 1 & 20 & 15 & 380 & 14 & 30 & 14 & 380 & 1 & 37 & 23 & 380 & \\
\hline 1 & 21 & 14 & 380 & 1 & 30 & 15 & 380 & 1 & 41 & 23 & 380 & \\
\hline 1 & 21 & 15 & 380 & 1 & 30 & 16 & 380 & 1 & 42 & 23 & 380 & \\
\hline 1 & 21 & 23 & 380 & 4 & 30 & 17 & 380 & 1 & 65 & 14 & 380 & $\sim 70$ \\
\hline 3 & 23 & 14 & 380 & 2 & 30 & 18 & 380 & 1 & 71 & 14 & 380 & $\sim 70$ \\
\hline 1 & 23 & 16 & 380 & 1 & 30 & 19 & 380 & 1 & 75 & 23 & 380 & $\sim 80$ \\
\hline 2 & 23 & 17 & 380 & 2 & 30 & 20 & 380 & 1 & 80 & 22 & 380 & $\sim 80$ \\
\hline 2 & 23 & 19 & 380 & 1 & 30 & 22 & 380 & 1 & 85 & 14 & 380 & $\sim 80$ \\
\hline 1 & 23 & 20 & 380 & 1 & 30 & 24 & 380 & 1 & 100 & 15 & 380 & $\sim 100$ \\
\hline 1 & 24 & 14 & 380 & 2 & 30 & 27 & 380 & 1 & 110 & 14 & 380 & $\sim 100$ \\
\hline 1 & 27 & 14 & 380 & 1 & 31 & 14 & 380 & 1 & 120 & 15 & 380 & $\sim 120$ \\
\hline 1 & 28 & 20 & 380 & 2 & 31 & 17 & 380 & 1 & - (150) & 21 & 380 & $\sim 150$ \\
\hline 1 & 29 & 8 & 380 & 1 & 31 & 20 & 380 & 1 & - & 17 & 380 & $\sim 165$ \\
\hline 3 & 29 & 14 & 380 & 1 & 31 & 21 & 380 & 1 & - & 16 & 380 & $\sim 250$ \\
\hline 1 & 29 & 15 & 380 & 1 & 31 & 30 & 380 & $1^{c}$ & 51 & 22 & 380 & $\sim 350$ \\
\hline 1 & 29 & 16 & 380 & 2 & 32 & 14 & 380 & 1 & - & 17 & 380 & $\sim 350$ \\
\hline 1 & 29 & 9 & 380 & 1 & 32 & 15 & 380 & 1 & - & 14 & 380 & $\sim 400$ \\
\hline 4 & 29 & 17 & 380 & 1 & 32 & 19 & 380 & 1 & - & 13 & 380 & $\sim 650$ \\
\hline
\end{tabular}

Number of triplets obtained for FMR1 and AFF2 genes, and ARX ex2p fragment size, in 100 male samples. The order is according to FMR1 allele sizes. Number of triplets obtained for FMR1 and AFF2 genes, and ARX ex2p fragment size, in 100 male samples, ordered according to FMR1 allele sizes. [n] Number of samples per allele profile; ${ }^{a} A F F 2$ intermediate size allele; ${ }^{b}$ control for the ARX c.429_452dup; ${ }^{c}$ FXS sample with mosaicism for an intermediate-size allele and a full mutation; Premutations clearly amplified by the multiplex-PCR and additionally confirmed by sequencing are bold highlighted; [-] not (reproducibly) amplified.

the multiplex-PCR, comparatively to Southern blotting, is the accuracy with which the allele-sizes are quantified, with emphasis in the clinically significant borderline alleles (Table 1).

In a sample belonging to a FXS patient (banked as a full mutation carrier) the multiplex-PCR assay revealed a fragment corresponding to a 51 CGG allele [Figure 1-I (d)]. Having excluded a sample identification error, another possible explanation for that finding, is that the blot (performed more than 16 years ago with an FMR1 specific ${ }^{32}$ P-labelled probe) may not have had sufficient sensitivity to pick up low levels of mosaicism or the expected intermediate band could have become imperceptible to the naked eye. With the aim of validating this result, an additional Southern blot analysis was performed in which, besides the full mutation, a faint intermediatesized band, was observed, consolidating the multiplexPCR result [Figure 2(a)]. This example illustrates that this assay or any other PCR-based methodology used as a screening test per se, is unable to exclude size-mosaicism. As such, it is recommended to exclude the presence of size-mosaics resorting to a different methodology (e.g. Southern blot), particularly in the presence of strong clinical features or FXS family history.

The validated multiplex-PCR assay was applied to 5000 samples (test group), previously screened for FMR1 expansions by Southern blot methods only. $64.4 \%$ of the analysed alleles were from male samples $(\mathrm{n}=3220)$ (Figure 3). In this subset, the FMR1 alleles ranged from 8 to 58 repeats, where the 54 CGG allele is the largest within the normal range, while $A F F 2$ alleles ranged from 6 to 68 CCGs, being the 44 repeat allele the largest among the normal. Alleles containing 29 CGG repeats and 14 CCG repeats were seen to be the most frequent, representing $35.0 \%$ and $33.1 \%$ of the FMR 1 and $A F F 2$ alleles respectively. FMR1 male intermediate-sized alleles (41 to 54 CGGs) revealed a frequency of $3.2 \%$, while for AFF2 gene only $0.71 \%$ fall into this category (31 to 44 CCGs). Although a direct comparison is difficult, these frequencies are comparable to those described in a similar survey focusing on the parameters used for the sample selection (using an inclusive rather than an exclusive selection) [9]. In a more recent study of a male subject control population, higher frequencies were obtained: 


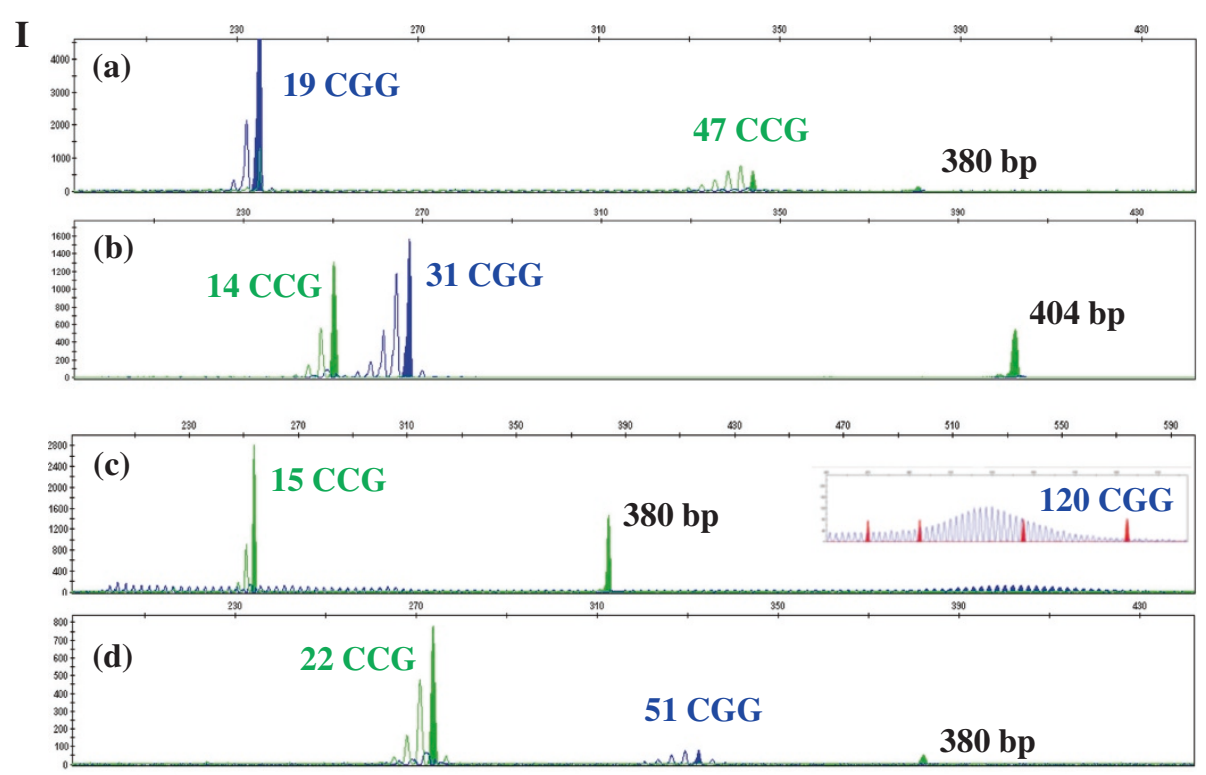

\section{II (a) FMRI - $110 \mathrm{CGG}$}

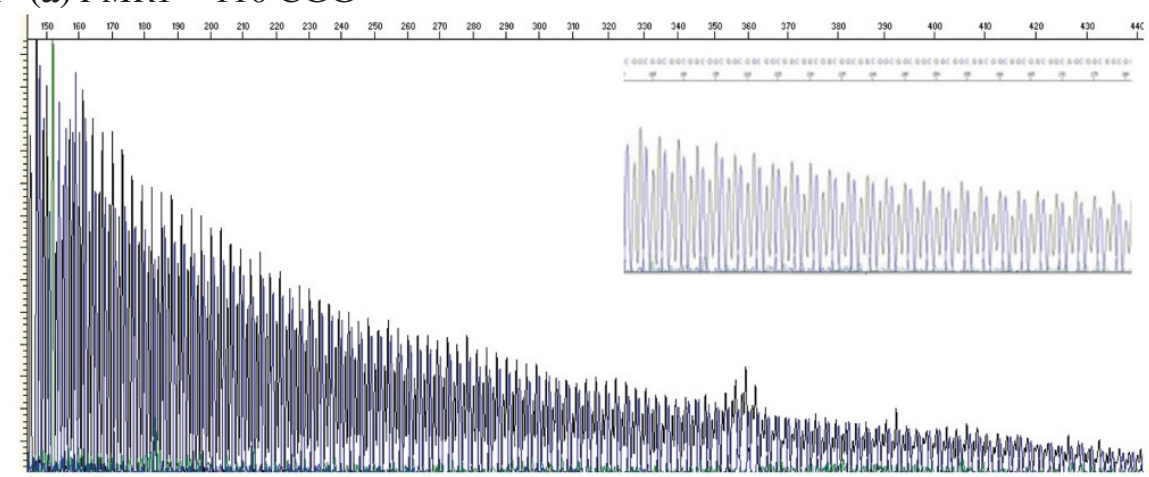

(b) $A F F 2-47$ CCG

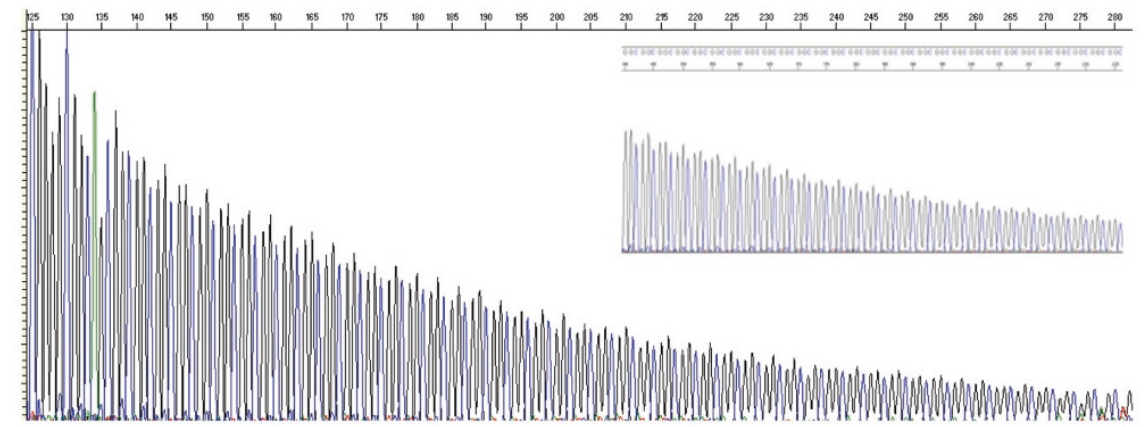

(c) $A R X-c .429 \_452$ dup

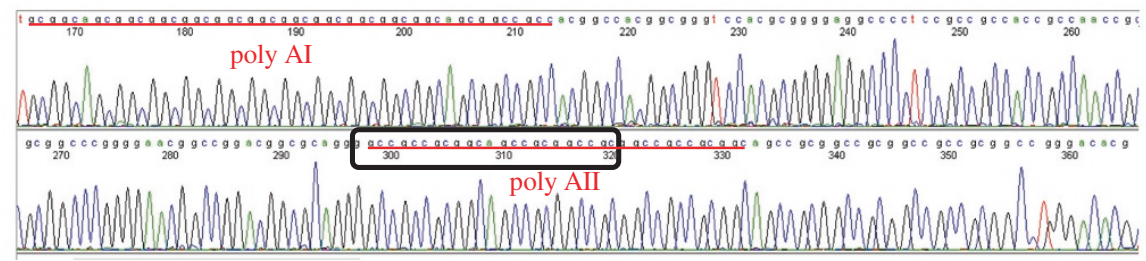

Figure 1 (See legend on next page.) 
(See figure on previous page.)

Figure 1 Examples of validation cohort results. I-Multiplex-PCR assay for the analysis of the mutational hotspot of FMR1 CGG alleles (blue label), AFF2 CCG alleles (green label) and ARX ex2p (380 bp green fragment). Profile: (a) AFF2 intermediate allele (47 CCGs); (b) c.429_452dup of ARX gene; (c) FMR1 premutation (120 CGGs); (d) FXS male mosaic with an FMR1 intermediate allele (51 CGGs) and a full mutation. II-Sequencing results: (a) CGG repetitive region of FMR1 gene (sense strand) showing the reproducibly sequenced premutated allele with 110 triplets; (b) AFF2 intermediate allele (47 CCGs) (reverse strand); (c) Partial nucleotide sequence of the ARX gene exon 2 (sense strand), showing the prevalent $A R X$ mutation c.429_452dup. The box indicates the most upstream twenty-four base pair sequence that is duplicated. The two stretches of repetitive triplets coding for alanine (poly Al and All) are underlined.

$6 \%$ for the FMR1 and $2 \%$ for the AFF2 intermediatesized alleles (Supplemental Table one in [22]). However, in that report a comparatively small number of samples were analysed $(\mathrm{n}=124)$.

Regarding the AFF2 gene, this multiplex-PCR assay revealed two presumed full mutations (2 in 3220), in which there was an unsuccessful amplification of the AFF2 allele and a case with an allele in the premutation range with 68 CCG repeats (1 in 3220) [Figure 2(g), Figure 3 and Figure 4(a)]. A definitive diagnosis of these cases requires a specific downstream technique; hence analyses by Southern blot using an AFF2 specific probe, as well as familial studies are being performed. The putative association of $A F F 2$ premutated alleles with the development of Parkinson disease in adulthood, the risk of expansion to pathogenic mutations in future generations as well as non-syndromic/non-pathognomonic clinical signs in AFF2 mutation carriers, justifies mutation screening for this gene and are obvious benefits of $A F F 2$ gene analysis included in this multiplex-PCR [22].

Co-amplification of $A F F 2$ and FMR1 has also proven useful in detecting $\mathrm{X}$-chromosome aneuploidy. In informative samples when the number of amplified fragments is inconsistent with the number of expected $\mathrm{X}$-chromosomes (according to patient gender) or there appears to be a discrepancy between the number of alleles for the different genes, further investigation must be done. In fact, we found nineteen of such cases in which the karyotype subsequently confirmed a Klinefelter syndrome, representing $0.59 \%$ of the analysed male samples, a frequency similar to others previously reported [Figure 2 (c), Figure 4(b) and (c)] [9]. Surprisingly, in a 47, XXY case, a fully-methylated FMR1 gene mutation was recently identified (data not shown).

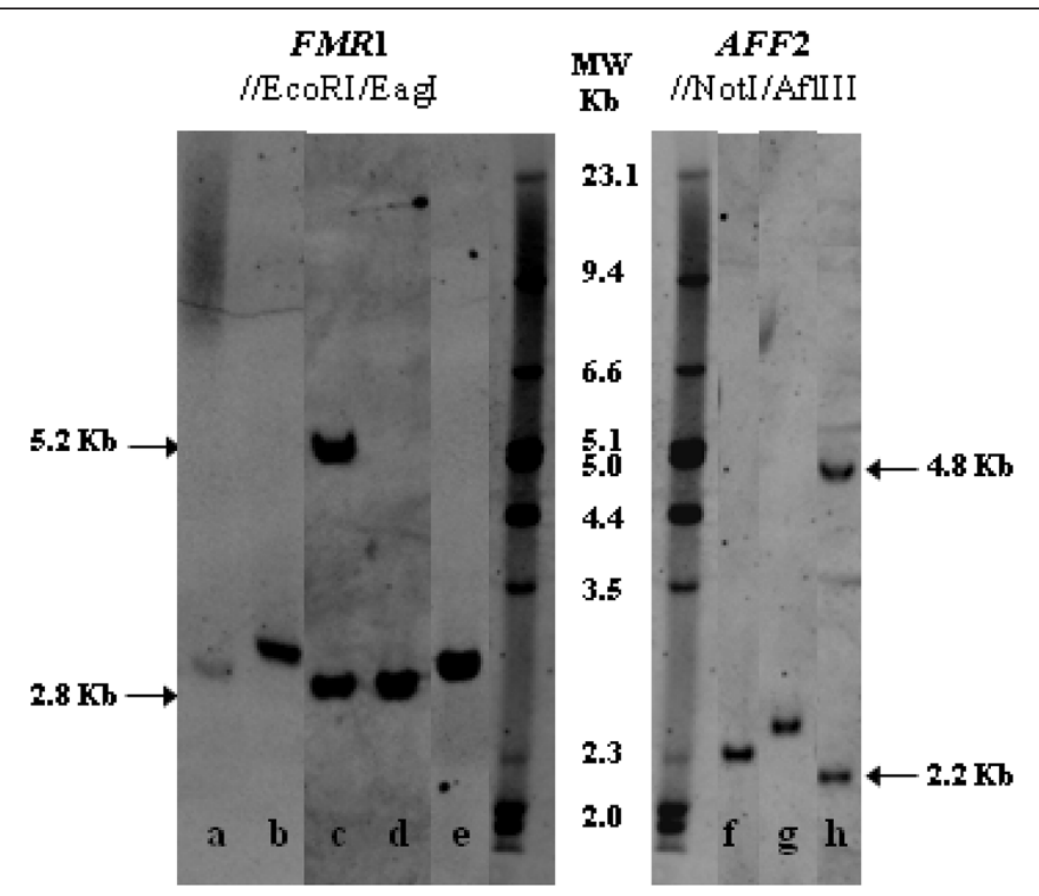

Figure 2 Examples of Southern blot results. Genotyping using FMR1 probe: After EcoRI /Eagl double digestion, normal females exhibit two fragments, a $\sim 2.8 \mathrm{~kb}$ (active X) and a $\sim 5.2 \mathrm{~kb}$ (inactive X), while in normal males only a $\sim 2.8 \mathrm{~kb}$ fragment is seen: (a) FXS male mosaic for a 51 CGG allele and a full mutation; (b) Premutated FMR1 male (110 CGGs); (c) Klinefelter with two FMR1 normal sized-alleles, showing the active and the inactive X-chromosome; (d) Normal FMR1 male (19 CGGs); (e) Premutated FMR1 male (58 CGGs). Genotyping using AFF2 probe: Following AflllI /Notl double digestion, normal females present two fragments, a $\sim 2.2 \mathrm{~kb}$ (active $\mathrm{X}$ ) and a $\sim 4.8 \mathrm{~kb}$ (inactive $\mathrm{X}$ ), whereas normal males exhibit only a 2.2 kb fragment: (f) Intermediate AFF2 male (47 CCGs); (g) Premutated AFF2 male (68 CCGs); (h) Homoallelic female [(CCG) $\left.{ }_{14} /(C C G)_{14}\right]$. 
In the male subset, thirteen size-variants were observed in the $A R X$ gene by fragment analysis, including seven deletions (ranging from 9 to $33 \mathrm{bp}$ ) and six insertions or duplications (represented by an increase in size of 3 to $24 \mathrm{bp}$ ) (Figure 3). Subsequent sequencing revealed four cases with the known $A R X$ mutation (c.429_452dup) as well as several other variants that change the alanine content of the ARX protein. Segregation studies and genotype/phenotype correlations are being completed. $A R X$ pathogenic variants characterized to date are the cause of at least ten clinically distinct conditions, where intellectual disability is the cardinal feature [10]. The vast majority of mutations occurring outside known ARX domains or in domains other than the polyalanine (poly A) tracks are 


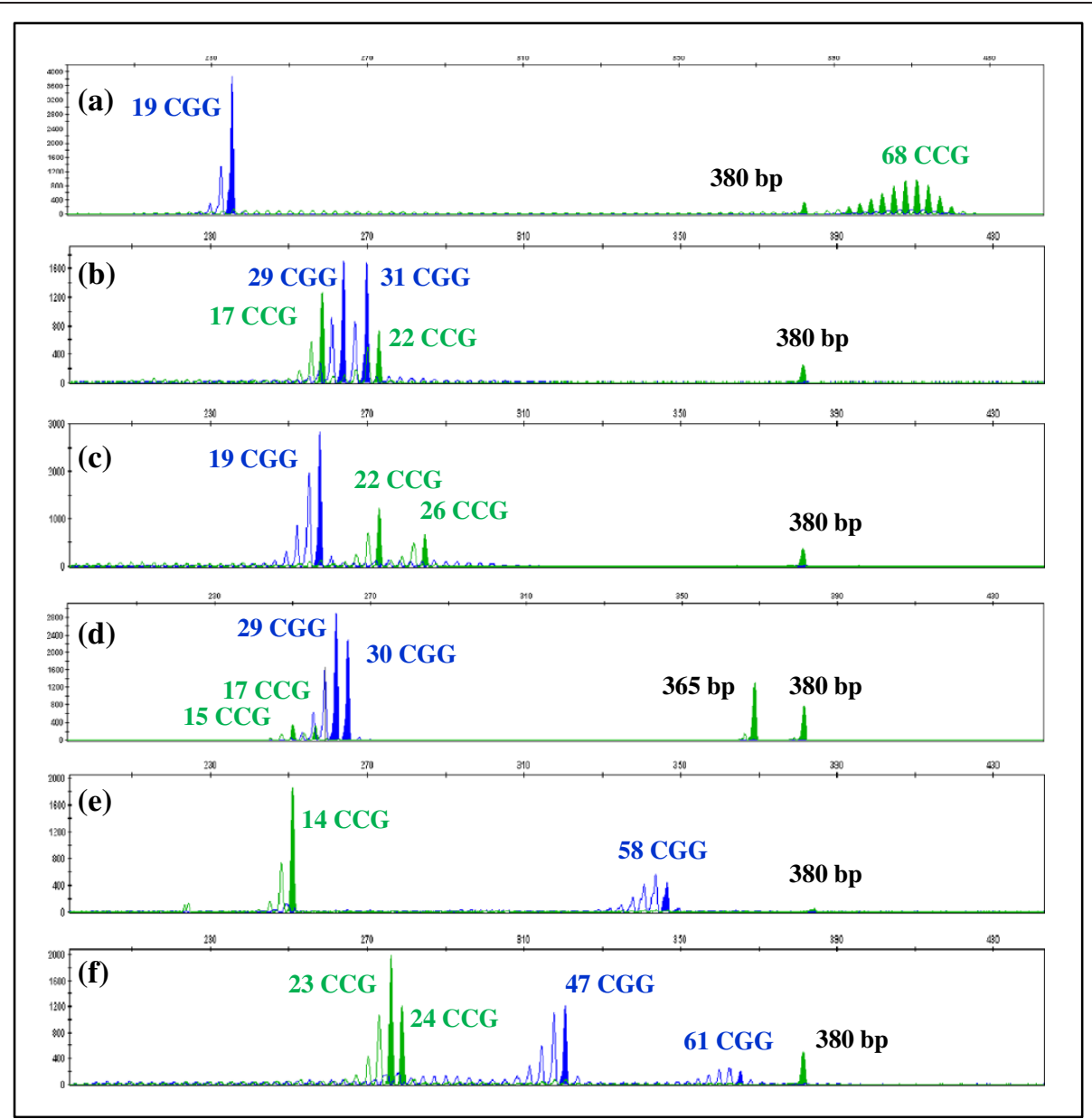

Figure 4 Examples of test group results. Multiplex-PCR assay focusing the analysis of the mutational hotspot of FMR1 CGG alleles (blue label), AFF2 CCG alleles (green label) and of the ARX ex2p (380 bp green fragment). Profiles: (a) AFF2 premutation male (68 CCGs); (b) and (c) male samples where the karyotype revealed the presence of an extra X-chromosome (47, XXY). Additionally in (c) an FMR1 full mutation was subsequently identified by Southern blotting. Profiles: (d) Heterozygous ARX exon 2 deletion female; (e) and (f): FMR1 premutation alleles, male (58 CGGs) and female $\left[(C G G)_{47} /(C G G)_{61}\right]$. Both FMR1 (d) and AFF2 (f) alleles differing in size by a single triplet can be easily distinguished.

known to cause the most severe forms of $A R X$ related disorders (e.g. X-linked lissencephaly with ambiguous genitalia). However, patients showing one or more of these particular features were not incorporated in the present survey. On the contrary, mutations that result in a poly A expansion account for $59 \%$ of $A R X$ mutations encountered in familial or isolated $A R X$-related cases [10]. Considering that our multiplex-PCR method is able to pinpoint deletions as well as expansions in the first two poly A stretches and that the latter are known to be the most frequent cause of $A R X$-related disorders, we can assume that the vast majority of the $A R X$-related variants of the test group male samples had been assessed.

In the females group, that represents $35.6 \%(\mathrm{n}=1780)$ of the analysed subjects, the allelic range of both FMR1 and AFF2 genes was similar to that obtained in male samples including several FMR1 or AFF2 intermediatesized alleles. Southern blot for the analysis of FMR1 or
AFF2 genes was carried out to discard a premutation or a full mutation in the case of an apparently homozygous women. Simultaneous FMR1 and AFF2 homoallelic samples were found in $5.8 \%$ of the female subjects $(\mathrm{n}=103)$ whereas private homoallelism for FMR1 or AFF2 genes, occurred respectively in $27.5 \%(\mathrm{n}=490)$ and $17.8 \%$ $(\mathrm{n}=317)$ of the samples [Figure 2(h)]. Considering the homoallelic females, the pairs [(CGG $\left.)_{29} /(\mathrm{CGG})_{29}\right]$ and $\left[(C C G)_{14} /(C C G)_{14}\right]$ were observed in $73.4 \%$ and $71.8 \%$ of samples, respectively. As such, the results obtained in these two subsets of samples were used for an overall representation of the female samples [Figure 5(a) and (b) respectively]. In the females group, the number of $A R X$ variants, was, as expected, significantly less than that observed in males: four size-variants of which three are deletions, ranging from 6 to $15 \mathrm{bp}$, and one is an insertion/ duplication of $24 \mathrm{bp}$. The sample with a $15 \mathrm{bp}$ deletion in $A R X$ exon 2 has the following allelic profile [(CGG) ${ }_{29} /$ 


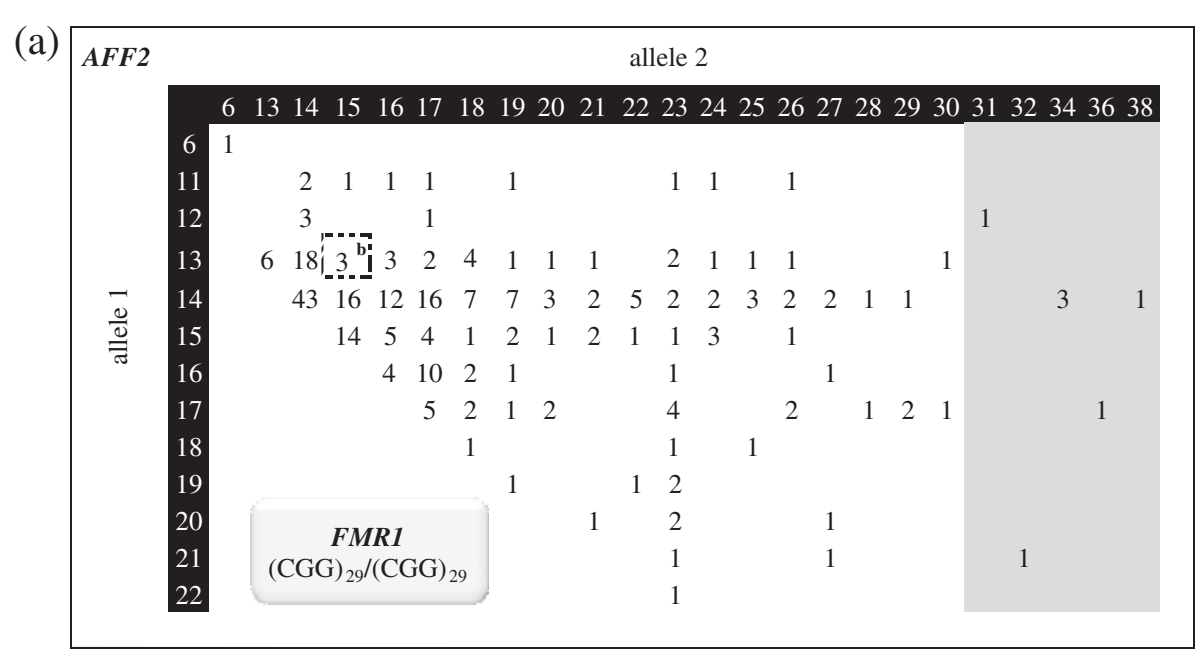

(b)

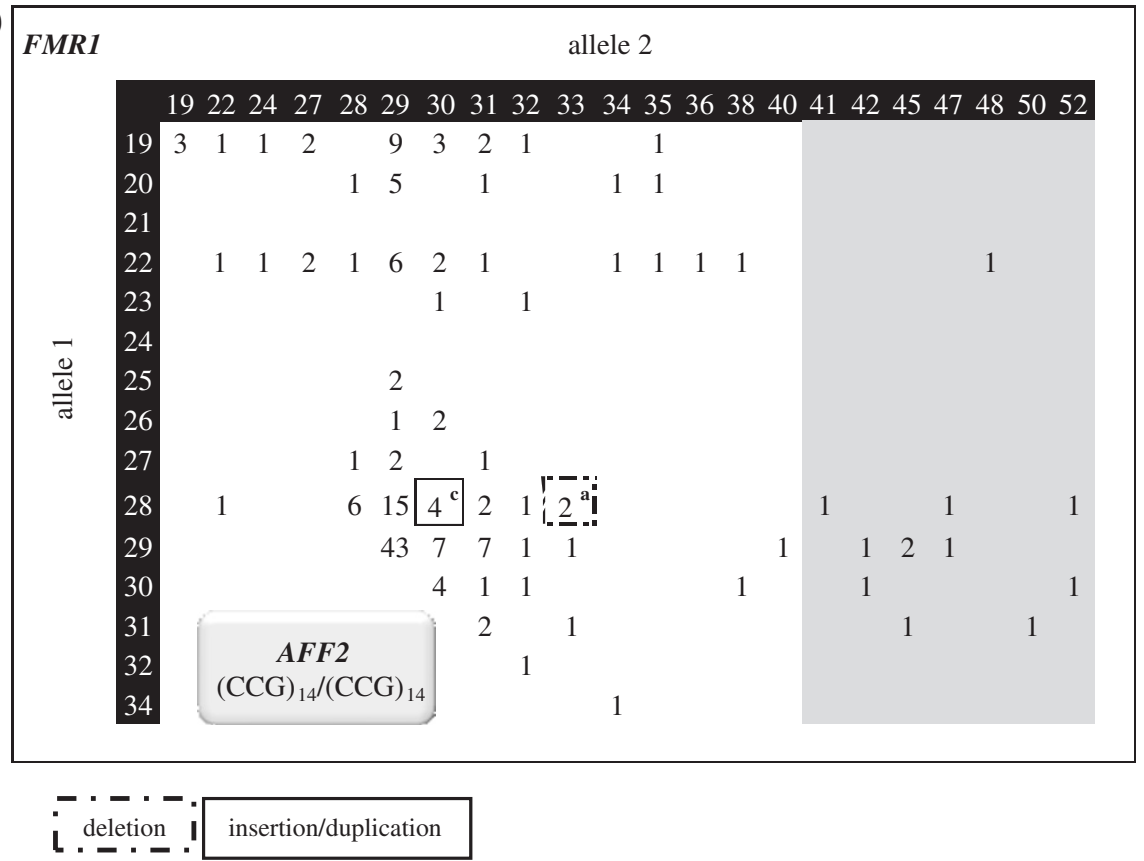

Figure 5 Test group results. Number of female homoallelic samples per allele profile of (a) FMR1 [(CGG) $\left.{ }_{29} /(C G G)_{29}\right]$ and (b) AFF2 [(CCG) ${ }_{14} /$ $\left.(C C G)_{14}\right]$. Intermediate alleles are grey shaded. Cases with different $A R X$ variants are surrounded by boxes. ARX variant type: ${ }^{a} 6$ bp deletion; ${ }^{b} 15$ bp deletion; ${ }^{c} 24$ bp insertion/duplication.

$\left.(\mathrm{CGG})_{30}\right]$ and $\left[(\mathrm{CCG})_{15} /(\mathrm{CCG})_{17}\right.$, while the remaining $A R X$ variants occurred in FMR1 or AFF2 homoallelic samples [Figure 4(d) and Figure 5(a) and (b)]. The pathogenic status of these variants, all occurring in a heterozygous state, remains to be determined.

Our test group includes subjects referred for FXS confirmation/exclusion, in the early 90s, classified as having a normal FMR1 profile (by Southern blot). However, two FMR1 premutations were now identified in a male (58 CGGs) and a female case $\left[(\mathrm{CGG})_{47} /(\mathrm{CGG})_{61}\right.$ ] [Figure 2 (e), Figure 3 and Figure 4(e) and (f)]. The blot was repeated using non-isotopic labelling, where these fragments were evident. We thus attributed the initial discrepancy to the relatively small size of the premutations, difficult to resolve under the older electrophoretic and labelling conditions. Despite Southern blotting and hybridization being the gold standard for molecular diagnosis of FXS and detection of mosaics, our results clearly illustrate the contribution of a PCR screening, particularly since it facilitates the detection and size-quantification of intermediate and premutated alleles.

The transition from an intermediate-sized allele to a full mutation in a single transmission was never observed, 
although changes in size can occur, particularly in female meiosis, which adds instability to those alleles [23]. The identification of these, probably unstable, alleles is of extreme importance since appropriate genetic counselling can be offered. Therefore, the benefits of the screening test are not limited to the subject tested but also are extended to other family members. Another added value concerns the FMR1 premutation carriers that, until recently, were not considered at risk for any clinical disorder, however nowadays the role of these alleles in terms of female reproduction, namely in fragile $\mathrm{X}$-associated primary ovarian insufficiency (FXPOI) cases and, in older individuals, in fragile X-associated tremor/ataxia syndrome (FXTAS) has been highlighted [24,25].

Based on our experience in FXS testing, one can estimate that around $3.5 \%$ of male and $\sim 1 \%$ of female samples will not amplify the FMR1 CGG repetitive region due to a mutation. Although unsuccessful amplification can be attributed to several factors (e.g. SNP in primer ligation site, deletion or technical pitfall) we do not have any such examples in our records as all these cases had a confirmed full mutation or large-premutation or both, confirmed by Southern blotting. Absence of amplification can occur due to the same factors described above in AFF2 or ARX testing. For these two genes no estimates can be made as a larger cohort, specifically referred due to intellectual disability, has to be analysed.

\section{Conclusion}

Overall, this multiplex-PCR assay can be beneficially used as a screening assay for FMR1 gene premutations under 110 CGGs, AFF2 gene premutations under 70 CCGs and $A R X$ exon 2 size-variants. This methodology allows the screening of a considerable number of male and female samples with the same sensitivity/specificity as the simplex methodology and an enhanced accuracy when compared to Southern blot. Based on our results, $0.031 \%$ of male sizemosaics might be unnoticed. In addition, this methodology will not detect very rare cases of point mutations or duplications/deletions outside the hotspots of the three genes. In females, this screening shall unravel $\sim 70 \%$ of $F M R 1$ and $\sim 80 \%$ of $A F F 2$ gene analysis. Final mandatory diagnosis of homoallelic cases, size and methylation mosaics, Xchromosome aneuploidies, or FMR1, AFF2 and ARX gene mutation are not aims of this methodology and must, thus, be achieved with other appropriate diagnostic techniques (e.g. Southern blotting, sequencing, methylationspecific PCR/MLPA or karyotyping). To overcome the putative overlap between the $A R X$ fragment and an AFF2 allele, an improvement would be the use of NED ${ }^{\text {ma }}$ labelling instead of HEX, in either the ARX or the AFF2 primer. Besides being rapid and cost-effective, this multiplex-PCR assay proved to be suitable as a high throughput screening strategy covering the previously mentioned XLID related conditions, representing a step forward in guiding the molecular approach and ultimately patient management, particularly in cases with difficult clinical ascertainment. This is definitely the case of $A R X$ gene mutations that include different mutational phenomena but also a clear hotspot that accounts for at least $60 \%$ of $A R X$ mutations [26,27]. In reports of patients with no obvious phenotype this number is usually increased. In fact, we found seventeen $A R X$ variants in our cohort of patients that showed no obvious phenotypic feature. In conclusion, laboratories performing molecular genetic studies of idiopathic intellectual disability would benefit from the implementation of this methodology, which enables a widened scope of detection of the mutational hotspot regions of FMR1, AFF2 and ARX genes in a single initial assay.

\section{Abbreviations}

$A R X$ ex2p: Portion of the second exon of ARX gene; bp: Base pairs; FRAXE: Fragile site "E", implicated in intellectual disability; FXPOI: Fragile Xassociated primary ovarian insufficiency; FXS: Fragile X syndrome; FXTAS: Fragile X-associated tremor/ataxia syndrome; gDNA: Genomic DNA; ID: Intellectual disability; MLPA: Multiplex ligation-dependent probe amplification; SNP: Single-nucleotide polymorphism; XLID: X-linked intellectual disability.

\section{Competing interests}

The authors declare that they have no competing interests.

\section{Authors' contributions}

PJ was responsible for the study design, data analysis and manuscript preparation; BO genotyped the vast majority of samples and contributed to the write-up of the manuscript; IM established and standardized the methodology, collaborated in data analysis and presentation of results; RS responsible for the overall study and critical review of the manuscript. All authors have read and approved the final manuscript.

\section{Acknowledgements}

The authors thank Manuela Vilarinho and Nuno Maia for outstanding technical assistance and Dr. M. Begoña Criado for her advice and support. The authors would also like to thank Dr. Francisco Martínez, from Hospital Universitario La Fe, Unidad de Genética y Diagnóstico Prenatal, Valencia, Spain for having provided a valuable sample, carrying the 24 bp duplication in ARX gene. The authors are greatful to ALFAGENE ${ }^{\circledR}$ Novas Tecnologias das Ciências da Vida, Lda., for support with publication costs.

\section{Author details}

${ }^{1}$ Centro de Genética Médica Dr. Jacinto Magalhães, CHP, Praça Pedro Nunes 88, 4099-028, Porto, Portugal. ${ }^{2}$ New address: Instituto Nacional de Saúde Dr. Ricardo Jorge INSA I.P., Lisbon, Portugal.

Received: 23 May 2012 Accepted: 7 June 2013

Published: 5 August 2013

\section{References}

1. Najmabadi H, Hu H, Garshasbi M, Zemojtel T, Abedini SS, Chen W, Hosseini M, Behjati F, Haas S, Jamali P, Zecha A, Mohseni M, Püttmann L, Vahid LN, Jensen C, Moheb LA, Bienek M, Larti F, Mueller I, Weissmann R, Darvish H, Wrogemann K, Hadavi V, Lipkowitz B, Esmaeeli-Nieh S, Wieczorek D, Kariminejad R, Firouzabadi SG, Cohen M, Fattahi Z, et al: Deep sequencing reveals 50 novel genes for recessive cognitive disorders. Nature 2011, 478:57-63.

2. Ropers HH, Hamel BC: X-linked mental retardation. Nat Rev Genet 2005, 6:46-57.

3. Chiurazzi P, Schwartz CE, Gecz J, Neri G: XLMR genes: update 2007. Eur J Hum Genet 2008, 16:422-434. 
4. Delbridge ML, McMillan DA, Doherty RJ, Deakin JE, Graves JA: Origin and evolution of candidate mental retardation genes on the human $\mathrm{X}$ chromosome (MRX). BMC Genomics 2008, 9:65-70.

5. Verkerk AJMH, Pieretti M, Sutcliffe JS, Fu Y-H, Kuhl DPA, Pizzuti A, Reiner O, Richards S, Victoria MF, Zhang F, Eussen BE, van Ommen G-JB, Blonden LAJ, Riggins GJ, Chastain JL, Kunst CB, Galjaard H, Caskey CT, Nelson DL, Oostra BA, Warren ST: Identification of a gene (FMR-1) containing a [CGG] repeat coincident with a break point cluster region exhibiting length variation in fragile X syndrome. Cell 1991, 65:905-914.

6. Crawford DC, Acuna JM, Sherman SL: FMR1 and the fragile $X$ syndrome: human genome epidemiology review. Genet Med 2001, 3:359-371.

7. Maddalena A, Richards CS, McGinniss MJ, Brothman A, Desnick RJ, Grier RE, Hirsch B, Jacky P, McDowell GA, Popovich B, Watson M, Wolff DJ: Technical standards and guidelines for fragile $X$ : the first of a series of diseasespecific supplements to the standards and guidelines for clinical genetics laboratories of the American college of medical genetics. Quality assurance subcommittee of the laboratory practice committee. Genet Med 2001, 3(3):200-205.

8. Bensaid M, Melko M, Bechara EG, Davidovic L, Berretta A, Catania MV, Gecz J, Lalli E, Bardoni B: FRAXE-associated mental retardation protein (AFF2) is an RNA-binding protein with high affinity for G-quartet RNA forming structure. Nucleic Acids Res 2009, 37:1269-1279.

9. Youings SA, Murray A, Dennis N, Ennis S, Lewis C, McKechnie N, Pound M, Sharrock A, Jacobs P: FRAXA and FRAXE: the results of a five year survey. J Med Genet 2000, 37:415-421.

10. Shoubridge C, Fullston T, Gécz J: $A R X$ spectrum disorders: making inroads into the molecular pathology. Hum Mutat 2010, 31:889-900.

11. Laperuta C, Spizzichino L, D'Adamo P, Monfregola J, Maiorino A, D'Eustacchio A, Ventruto V, Neri G, D'Urso M, Chiurazzi P, Ursini MV, Miano MG: MRX87 family with Aristaless X dup24bp mutation and implication for polyalanine expansions. BMC Med Genet 2007, 8:25-29.

12. Suri M: The phenotypic spectrum of $A R X$ mutations. Dev Med Child Neurol 2005, 47:133-137.

13. Shoubridge C, Gardner A, Schwartz CE, Hackett A, Field M, Gécz J: Is there a Mendelian transmission ratio distortion of the C.429_452(24bp) polyalanine tract ARX mutation? Eur J Hum Genet 2012, 20(12):1311-1314.

14. van Karnebeek CD, Jansweijer MC, Leenders AG, Offringa M, Hennekam RC: Diagnostic investigations in individuals with mental retardation: a systematic literature review of their usefulness. Eur J Hum Genet 2005, 13:6-25.

15. Miller SA, Dykes DD, Polesky HF: A simple salting out procedure for extracting DNA from human nucleated cells. Nucleic Acids Res 1988, 16(3):1215.

16. Rousseau F, Heitz D, Biancalana V, Blumenfeld S, Kretz C, Boue J, Tommerup N, Van Der Hagen C, DeLozier-Blanchet C, Croquette MF, Gilgenkrantz S, Jalbert P, Voelckel MA, Oberlé I, Mandel JL: Direct diagnosis by DNA analysis of the fragile $X$ syndrome of mental retardation. N Engl J Med 1991, 325:1673-1681.

17. Romero-Rubio MT, Andrés-Celma M, Castelló-Pomares ML, Roselló M, FerrerBolufer I, Martínez-Castellano F: ARX mutations and mental retardation of unknown etiology: three new cases in Spain. Rev Neurol 2008, 47(12):634-637.

18. Nahhas FA, Monroe TJ, Prior TW, Botma PI, Fang J, Snyder PJ, Talbott SL, Feldman GL: Evaluation of the human fragile $\mathrm{X}$ mental retardation 1 polymerase chain reaction reagents to amplify the FMR1 gene: testing in a clinical diagnostic laboratory. Genet Test Mol Biomarkers 2012, 16(3):187-192.

19. Khaniani MS, Kalitsis P, Burgess T, Slater HR: An improved Diagnostic PCR Assay for identification of Cryptic Heterozygosity for CGG Triplet Repeat Alleles in the Fragile X Gene (FMR1). Mol Cytogenet 2008, 1:5.

20. Lyon E, Laver T, Yu P, Jama M, Young K, Zoccoli M, Marlowe N: A simple, highthroughput assay for Fragile $X$ expanded alleles using triple repeat primed PCR and capillary electrophoresis. J Mol Diagn 2010, 12(4):505-511.

21. Elias $M H$, Ankathil $R$, Salmi AR, Sudhikaran W, Limprasert P, Bin Alwi Zilfalil BA: A New Method for FMR1 Gene Methylation Screening by Multiplex Methylation-Specific Real-Time Polymerase Chain Reaction. J Mol Diagn 2011, 15(6):387-393

22. Costa A, Gao L, Carrillo F, Cáceres-Redondo MT, Carballo M, Díaz-Martín J, Gómez-Garre P, Sobrino F, Lucas M, López-Barneo J, Mir P, Pintado E: Intermediate alleles at the FRAXA and FRAXE loci in Parkinson's disease. Parkinsonism Relat Disord 2011, 17(4):281-284.

23. Hantash FM, Goos DN, Crossley B, Anderson B, Zhang K, Sun W, Strom CM: FMR1 premutation carrier frequency in patients undergoing routine population-based carrier screening: insights into the prevalence of fragile $X$ syndrome, fragile $X$-associated tremor/ataxia syndrome, and fragile $X$-associated primary ovarian insufficiency in the United States. Genet Med 2011, 13:39-45.

24. Seixas Al, Vale J, Jorge P, Marques I, Santos R, Alonso I, Fortuna AM, PintoBasto J, Coutinho P, Margolis RL, Sequeiros J, Silveira I: FXTAS is rare among Portuguese patients with movement disorders: FMR1 premutations may be associated with a wider spectrum of phenotypes. Behav Brain Funct 2011, 7:19.

25. Miano MG, Laperuta C, Chiurazzi P, D'Urso M, Ursini MV: Ovarian dysfunction and FMR1 alleles in a large Italian family with POF and FRAXA disorders: case report. BMC Med Genet 2007, 11:8-18.

26. Shinozaki Y, Osawa M, Sakuma H, Komaki H, Nakagawa E, Sugai K, Sasaki M, Goto Y: Expansion of the first polyalanine tract of the ARX gene in a boy presenting with generalized dystonia in the absence of infantile spasms. Brain Dev 2009, 31(6):469-472.

27. Cossée M, Faivre L, Philippe C, Hichri $H$, de Saint-Martin A, Laugel V, BahiBuisson N, Lemaitre JF, Leheup B, Delobel B, Demeer B, Poirier K, Biancalana V, Pinoit JM, Julia S, Chelly J, Devys D, Mandel JL: ARX polyalanine expansions are highly implicated in familial cases of mental retardation with infantile epilepsy and/or hand dystonia. Am J Med Genet A 2011, 155A(1):98-105.

doi:10.1186/1471-2350-14-80

Cite this article as: Jorge et al:: Development and validation of a multiplex-PCR assay for X-linked intellectual disability. BMC Medical Genetics 2013 14:80.

\section{Submit your next manuscript to BioMed Central and take full advantage of:}

- Convenient online submission

- Thorough peer review

- No space constraints or color figure charges

- Immediate publication on acceptance

- Inclusion in PubMed, CAS, Scopus and Google Scholar

- Research which is freely available for redistribution

Submit your manuscript at www.biomedcentral.com/submit
C Biomed Central 